\title{
TRATAMENTO DE FRATURA ISOLADA DO ARCO ZIGOMÁTICO - RELATO DE CASO
}

Treatment of isolated fracture of the zygomatic arch - case report

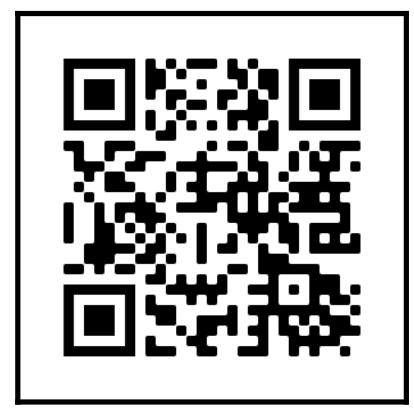

Autores:

Antônio José Araújo Pereira Júnior

Staff do Departamento de Cirurgia Maxilofacial do Hospital Regional de Barbacena Dr. José Américo/Fundação Hospitalar do Estado de Minas Gerais (FHEMIG) - MG

\section{Isabela Possas da Fonseca Pereira}

Staff do Departamento de Cirurgia Maxilofacial do Hospital Regional de Barbacena Dr. José Américo/Fundação Hospitalar do Estado de Minas Gerais (FHEMIG) - MG

\section{Haniel Henrique Dutra Clemente}

Graduando em Odontologia pela Universidade Federal de Juiz de Fora (UFJF) - MG

\section{Heloísa de Souza Rodrigues}

Graduando em Odontologia pela Universidade Federal de Juiz de Fora (UFJF) - MG

Categoria da seção: Relato de caso

\section{Endereço para correspondência:}

Antônio José Araújo Pereira Júnior

Avenida Luz Interior, 105 - Bairro Estrela Sul - Juiz de Fora - MG; CEP 36030-776;

Telefone: +55 (32)98876-3260;

E-mail:_apereirabucomaxilo@yahoo.com.br 


\section{RESUMO}

Devido ao comprometimento estético funcional, as fraturas do arco zigomático (AZ) devem ser abordadas, o mais breve possível, com o objetivo de minimizar as sequelas. Há várias formas de tratamento descritas na literatura. $\mathrm{O}$ objetivo deste trabalho é relatar um caso clínico de fratura isolada do $A Z$ tratada pela técnica de Keen sob anestesia local.

Palavras-chave: Arco zigomático; Fraturas; Terapêutica.

\section{ABSTRACT}

Due to functional aesthetic impairment, fractures of the zygomatic arch (ZA) should be addressed, as soon as possible, in order to minimize the sequelae. There are several forms of treatment described in the literature. The objective of this work is to report a clinical case of isolated ZA fracture treated by Keen's technique under local anesthesia.

Key words: Zygomatic arch; Fractures; Therapy.

\section{INTRODUÇÃO}

A ruptura do tecido ósseo com consequente solução de continuidade denomina-se fratura óssea. $O$ tipo e a extensão de uma fratura de face dependem de vários fatores, como a anatomia da região, a direção e a força do impacto (PRADO et al, 2004).

Fraturas isoladas do arco zigomático (AZ), sem outras lesões que envolvam o complexo zigomático-maxilar, correspondem a menos de $10 \%$ das lesões zigomáticas, e ocorrem quando uma força é aplicada diretamente a partir da face lateral da cabeça (FONSECA et al, 2015).

Clinicamente, o paciente pode apresentar dor, trismo, depressão no tecido mole na região do arco zigomático, escoriações, edema ou hematoma sobre o local traumatizado (PRADO et al, 2004; KORKMAZ et al, 2016; OLIVEIRA, 2009).

O diagnóstico imaginológico pode ser feito através de radiografias extraorais (projeção Submento-vértice (Hirtz) e Mento-naso (Waters)) ou tomografia computadorizada (TC), sendo essa o padrão-ouro (MILORO et al, 2008).

As fraturas do arco podem ser divididas em dois grupos: 1) fratura isolada do arco; 2) fratura combinada na qual o AZ se rompeu com outros ossos da face. As fraturas isoladas são divididas em dois subgrupos: a) 02 fraturas no arco; b) 
mais de 02 fraturas ou em forma de V. Já as fraturas combinadas, elas foram subgrupadas em: a) fratura única do arco; b) fratura plural (OZYAZGAN et al, 2007).

Diferentes técnicas e dispositivos têm sido descritos, por muitos autores, a respeito do tratamento das fraturas do arco zigomático. Entre as principais estão: 1) Abordagem temporal de Gillies; 2) Abordagem intraoral de Keen; 3) Redução percutânea; 4) Redução aberta com fixação interna (FONSECA et al, 2015; ROBIONY et al, 2012; GIUDICE et al, 2020).

O intuito deste artigo é demostrar um caso de tratamento, em ambiente emergencial, de fratura isolado do arco zigomático esquerdo por meio da Técnica de Keen sob anestesia local.

\section{APRESENTAÇÃO DO CASO}

Paciente do sexo masculino, 34 anos, vítima de agressão física, procurou o serviço de Cirurgia e Traumatologia Bucomaxilofacial do Hospital Regional de Barbacena Dr. José Américo - MG, apresentando limitação em abertura bucal, edema, depressão e sensibilidade na região do $A Z$ esquerdo à palpação (Figura 1). A fratura foi confirmada por uma tomografia computadorizada (Figura 2), sendo o paciente submetido, imediatamente, à redução da fratura sob anestesia local através da abordagem intraoral de Keen (incisão no sulco vestibular superior, abaixo do pilar zigomático; e redução do AZ com elevador curvo), com resultado estético-funcional satisfatório (Figura 3).

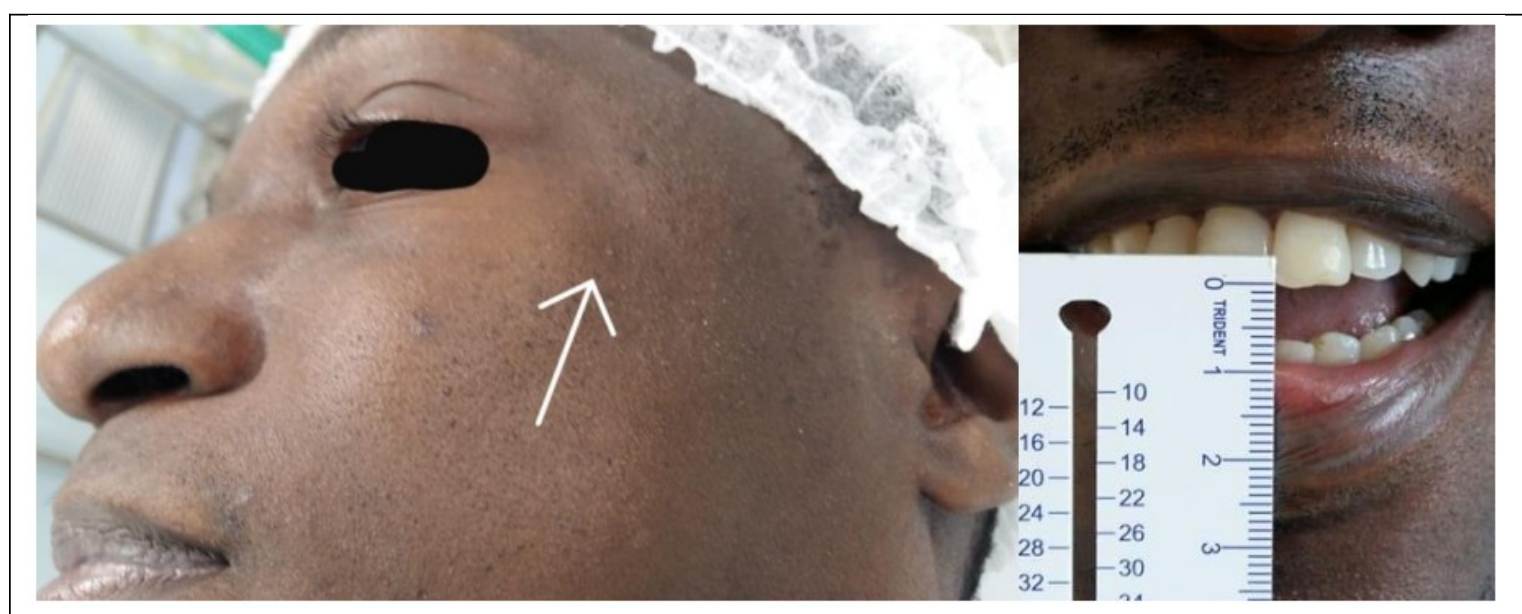

Figura 1 - Aspecto clínico evidenciando a depressão e a limitação de abertura bucal. 


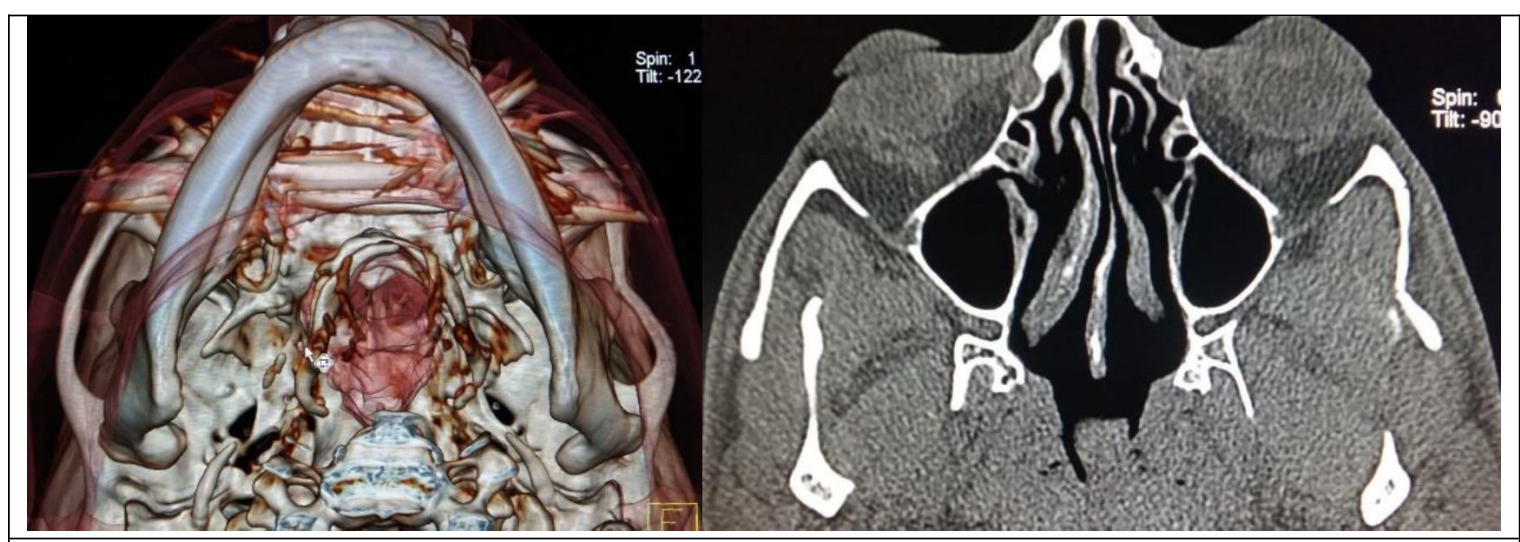

Figura 2 - TC de face demonstrando a fratura isolada do arco zigomático esquerdo.

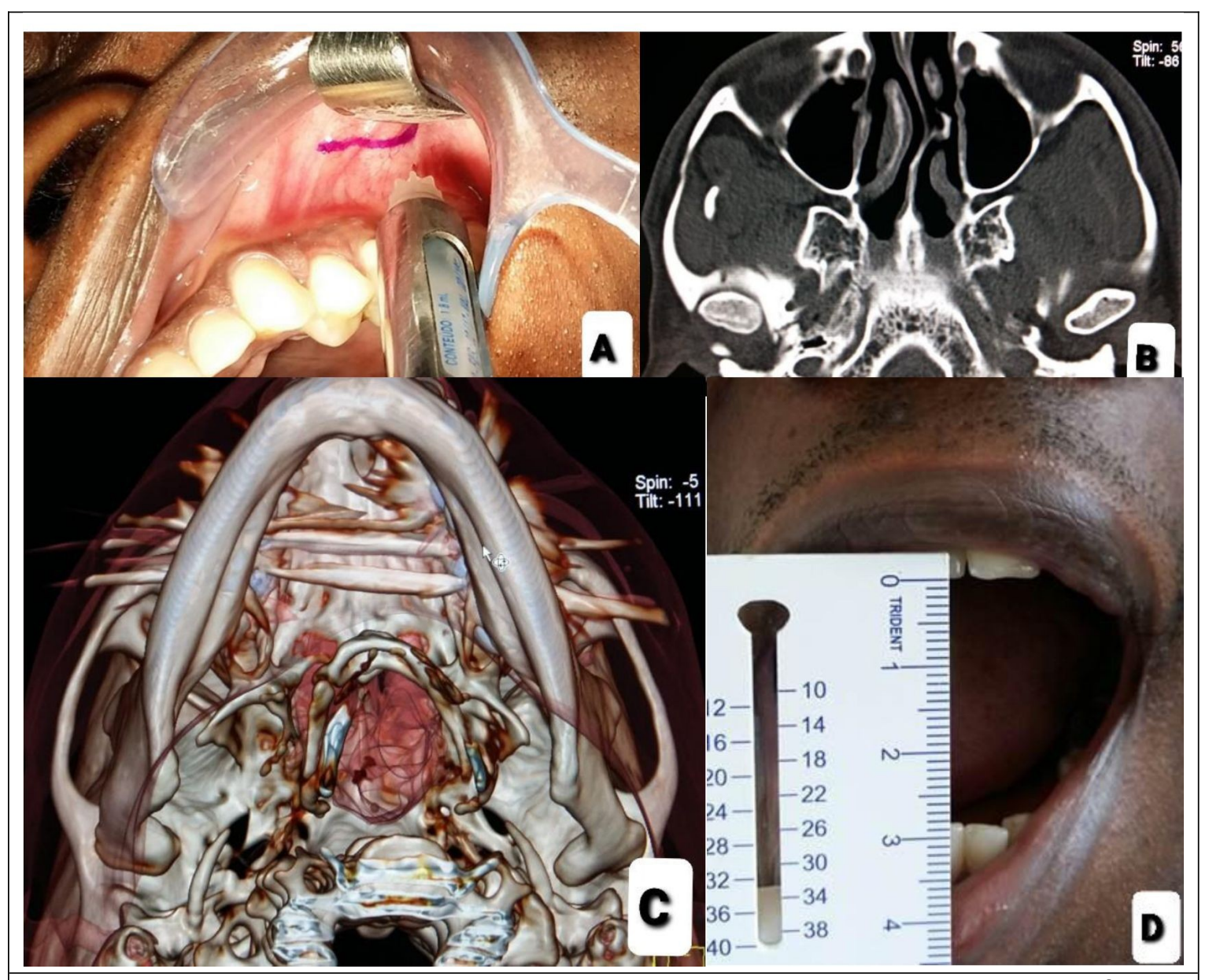

Figura 3 -A)Abordagem intraoral de Keen sob anestesia local; B) Redução estável da fratura em TC axial; C) Aspecto 3D do arco zigomático esquerdo; D) Melhora significativa da abertura bucal no pós operatório imediato

\section{DISCUSSÃO}

Usualmente, as fraturas do AZ não requerem qualquer método de fixação após 
sua correta redução, uma vez que se mantém estáveis devido, muitas vezes, à ação da aponeurose do músculo temporal que aí se insere. Em fraturas instáveis ou deslocadas grosseiramente, a técnica de osteossíntese com uso de placa e parafuso mostrou-se um procedimento seguro e estável (OLIVEIRA et al, 2009).

A redução das fraturas do $A Z$ pode ser realizada por qualquer das técnicas descritas na literatura. Um gancho ósseo percutâneo, a abordagem temporal de Gillies e a intraoral de Keen, todas são técnicas aceitáveis. A necessidade de estabilizar fraturas do $A Z$ varia com a localização da lesão, o número de fraturas e o deslocamento dos segmentos (FONSECA et al, 2015).

Vários autores (KORKMAZ et al, 2016; ROBIONY et al, 2012) afirmam que a abordagem intraoral, para restauração estético-funcional das deformidades do arco zigomático pós-traumático, é vista como um método simples, seguro e econômico, pois pode ser realizado sob anestesia local e não necessita de hospitalização pós-operatória, corroborando com o caso clínico exposto neste artigo.

\section{CONCLUSÕES}

A redução de fraturas isoladas do arco zigomático através da técnica de keen é simples, rápida, sem complicações significativas e com ótimos resultados. Além disso, permite que seja realiza em ambiente ambulatorial sob anestesia local.

\section{REFERÊNCIAS BIBLIOGRÁFICAS}

1. Prado R, Salim MAA. Cirurgia bucomaxilofacial - Diagnóstico e tratamento. 1 ed., Guanabara Koogan, 2004. p. 457-508.

2. Fonseca JR, Walker RV, Barber HD, Powers MP, Frost DE. Trauma Bucomaxilofacial. 4 ed., Elsevier, 2015. p. 828-846.

3. Korkmaz YT, Coskun U, Durmuslar MC, Zor ZF, Hocaoglu TP, Altintas NY. Reduction of isolated zygomatic arch fractures using dental instrument: reporto 2 cases and review of literature. J Pak Med Assoc. 2016; 66(3): 345-347.

4. Oliveira JAGP. Fratura do arco zigomático: relato de caso utilizando o acesso pré- auricular. Rev Cir Traumatol Buco-Maxilo-Fac. 2009; 9(1): 
47-52.

5. Miloro M, Ghali GE, Larsen PE, Waite PD. Princípios de cirurgia bucomaxilofacial de Peterson (volume 1). 1 ed., Santos, 2008. p. 445-462.

6. Ozyazgan I, Günay GK, Eskitascioglu T, Ozkose M, Coruh A. A new proposal of classification of zygomatic arch fractures. J Oral Maxillofac Surg. 2007; 65(3): 462469.

7. Robiony M, Tenani G, Bellini P, Salgarelli AC. Intraoral approach for aesthetic restoration of posttraumatic zygomatic arch deformities. J Craniofac Surg. 2012; 23(5): 1418-1420.

8. Giudice A, Cristofaro MG, De Cicco D, Barca I, Dell Aversana Orabona G, Califano L. Percutaneous reduction of isolated zygomatic arch fractures: A 5-year experience. J Oral Maxillofac Surg. 2020; doi: 10.1016/j.joms.2020.02.002. 